\title{
Nutritional factors in etiology of childhood obesity
}

\author{
Onur Çirak ${ }^{1 *}$, Hacı Ömer Yilmaz² and Nurcan Yabancı Ayhan ${ }^{3}$ \\ ${ }^{1}$ Student of PhD., Faculty of Health Sciences, Ankara University, Ankara, Turkey \\ ${ }^{2}$ Student of PhD., Faculty of Health Sciences, Gümüşhane University, Gümüşhane, 29000, Turkey \\ ${ }^{3}$ Professor, Faculty of Health Sciences, Ankara University, Ankara, Turkey
}

\begin{abstract}
One of the most important factors in childhood obesity is nutrition. There are a lot of factors that also effect nutrition status and behaviours of children. Factors such as the historical process of food consumption, changes in available foods, inability to manage energy intake, nutritional behaviors of the family, food types that kept at home, maternal nutritional status during pregnancy and breastfeeding are all closely related to obesity in children. In this review, it was aimed to examine the effect of nutritional factors on etiology of childhood obesity. In order to prevent and treat obesity both nutritional status and factors effecting it should be taken into consideration. Childhood obesity is not easy to treat and to improve treatment and success rate, new nutritional approaches should be developed. Dietitians have a major role in nutritional status, nutritional behaviors of children and diagnosis, treatment and monitoring of childhood obesity.
\end{abstract}

\section{Introduction}

As of the 21st century, obesity, which has become a global health problem, has also become widespread in children. Childhood obesity, which requires a complex and versatile approach, has been an important issue for healthcare workers due to the lack of an easy solution [1]. And nutritional problems faced by children and adolescents related to nutrition are similar across the world [2].

The World Health Organization (WHO) states that most boys and girls in developing countries enter adolescence with undernourishment, as well as a growing number of mildly obese and obese children and adolescents in both low and high-income countries [3]. Being overweight affects the social life, physiological state and psychological health of the child in the long term [4]. Aside from the health problems associated with childhood obesity are, social and environmental problems patients face should be taken into consideration during treatment process [5]. Healthy eating and proper nutritional behaviors positively affect children's body composition and health and reduce cognitive stress and psychological problems [6]. Increase in overweight and obesity prevalence among children and adolescents is an important community health problem and it is related to nutritional behavioral factors. [7].

In this review, it was aimed to examine the effect of nutritional factors on etiology of childhood obesity.

\section{Childhood obesity}

In the assessment of obesity in children and adolescent populations, BMI (Body Mass Index) is used as a screening tool but not for diagnostic purposes. In children aged 2 to 20 years, body composition evaluated according to the CDC (Centers for Disease Control and Prevention) percentile values of BMI for age and BMI for sex. If BMI percentile value for age is between 85-95 the child is considered as "overweight" and if BMI value is over 95 it is considered as "obese" [8]. As a result of a study of obesity in 634 children aged 6-12 years, it was found that $8.8 \%$ of the children were overweight and $3.1 \%$ were obese and that obesity was associated with using transportation vehicles, fast food intake, inadequate physical activity and low fruit and vegetable intake [9].

In our country (Turkey), different reference values are used to assess obesity frequency in children and adolescents. According to results of Surveillance on Growth Monitoring in School Aged Children in Turkey (TOÇBİ, 2011) the overweight and obesity rates of children 6-10 years old are $14.3 \%$ and $6.5 \%$, respectively [10]. As a result of Childhood Obesity Surveillance Initiative in Turkey (COSI-TUR, 2016), overweight and obesity rates were found as $14.6 \%$ and $9.9 \%$ in children between 7-8 years, respectively [11].

In a study, nutritional behavior and physical activity of 800 overweight and obese children (10-14 years) were investigated. It was determined that $20.4 \%$ of males were overweight, $10.3 \%$ of them were obese, of females $9.7 \%$ were overweight, and $22.5 \%$ were overweight. Obesity prevalence was found to be significantly higher in girls and at younger ages. In addition, skipping the main meals especially in the case of breakfast skipping or long time between meals, extra snacks consumption, irregular eating behaviors and the increase in outside food consumption were found to have a significant relationship with being overweight and obesity. Obesity prevalence was found to be significantly higher in children who never consume green vegetables or fruits, daily consume soft drinks, bakery products, chocolate, fried foods. Also $26.6 \%$ of children were found to be sedentary, and physical activity level was significantly correlated with obesity prevalence [7].

In addition, in children younger than 2 years, the World Health Organization (WHO) offers guidelines for the head circumference, height, body weight, and suggests the use of these guidelines. And children with measured values 98 th percentile and above are considered

${ }^{*}$ Correspondence to: Onur ÇIRAK, Student of PhD, Faculty of Health Sciences, Ankara University, Ankara-06000, Turkey, Email: onrcrk@hotmail.com

Key words: Childhood obesity, Etiology, Nutrition, Nutritional Factors

Received: July 11, 2018; Accepted: July 19, 2018; Published: July 24, 2018 
obese [8]. It is known that obese children are tending to be obese adults, in addition to that BMI assessment should be done correctly and children should not be diagnosed with obesity by mistake. For this, it is also necessary to determine whether the excess weight of the child is really caused by fat tissue or muscle tissue. The amount of muscle and fat tissue of children varies according to sex and ethnicity [5-13].

Identification of nutritional status of children and adolescents, especially in developing countries, has a great importance in the upbringing of healthy individuals [14]. When nutritional status of children and adolescents is evaluated, body weights and height lengths should be measured; weight loss and gain, growth retardation, physical activity should be recorded; the number of daily meals and the foods consumed at these meals should be evaluated, sexual development and girls' menstruation history should be taken [15].

Many nutrition related health problems, such as hypertension, type 2 diabetes, dyslipidemia, insulin resistance, sleep apnea, asthma, and non-alcoholic liver fatigue used to occur only in adults. However, nowadays these nutrition related health problems are common in children due to obesity which is also a nutrition related health problem. However, the problems caused by the obesity in pediatric cases are not limited to physical and physiological. These children also face psychological problems such as depression, anxiety, lack of self-confidence, and sometimes eating disorders [5-12]. Obesity has a greater association with many chronic diseases than with hunger, poverty, smoking and alcohol use [5].

\section{Nutritional factors in etiology of childhood obesity}

Adequate and balanced nutrition for children and adolescents is important; but in this period undesirable nutritional behaviors such as skipping meals and applying the wrong diet can be observed. It is anticipated that nutritional behavior is related to nutrition information and that individuals educated on healthy nutrition will have healthy nutritional practice [16].

In the treatment of childhood obesity, the main purpose of lifestyle modification practice is to change the body composition. However, it is not known exactly how much these practices will affect food consumption and energy intake [17]. Nutritional status and practiced diets are one of the most important causes of increase of obesity in children and adolescents. Factors such as the historical process of food consumption, changes in available foods, inability to manage energy intake, nutritional behaviors of the family, food types that kept at home, maternal nutritional status during pregnancy and breastfeeding are all closely related to obesity in children [1-18].

\section{The historical process of food consumption}

The prelatic period diets and modern-day diets are completely different from each other. In addition, inhabited life, the standing out of a sedentary lifestyle, easy access to foods, and especially the altered living conditions, such as the spread and consumption of processed foods, have led to serious changes in body composition and an increase in the prevalence of obesity in children [19].

The most important differences between the diets in the old times and modern-day diets are high pulp content, low energy content, containment of various nutrients and long chained fatty acids. In the paleotic period, the proportion of $n-6 / n-3$ fatty acids taken from the diet were 1: 1 , it is 15-17:1 nowadays [20]. In the earlier periods, eating practices based on vegetables and fruits, fish-lean meats and oil seeds were on the forefront of nutrition. As a result of settling and agricultural applications, nutritional practices transferred to a grainbased diet. Over time, vitamin mineral deficiencies and high-energy intake diets have come to the forefront. As a result, consumption of high-energy foods started to establish. In addition to this, the energy used for getting or accessing to foods today, is very low compared to the energy taken with foods [5-13].

\section{Changes in available foods}

Today, the foods served in restaurants and cafes are high in energy, salt, sugar and fat content, and low in pulp. Also including children's menus, portions of served foods are large in these menus. All of these are associated with childhood obesity. In addition to these, despite the increased production of energy-rich foods such as various fast foods, snacks, sweets, energy needs of children remain constant. For this reason, consumption of these foods is related to obesity during childhood as well [19].

In a study, fast food consumption and obesity rates in 201 (12-18 years) adolescents were aimed to evaluate. And it was found that $19 \%$ of adolescents were mildly obese and consumed fast food $1.5 \pm 1.33$ times per week. As a result, fast food consumption was found to be an important nutritional factor in the occurrence and prevalence of obesity in adolescents [21].

One thousand six hundred twenty-six children (7-16 years) were included in a study which was conducted for assessment of fast food consumption, obesity and hypertension seen in children. Food consumption records and anthropometric measurements of the children were taken. According to the data collected, it was found that $11.1 \%$ of the children were obese, $19.7 \%$ of them had abdominal fat and 9.0\% had hypertension. Obesity prevalence was significantly higher in males than females and nearly half of males ( $51.9 \%$ of males and $43.6 \%$ of females) consumed fast food regularly during the last 3 months [22].

In a study conducted with 1349 overweight and obese children. And children were treated with a diet in which sugary drinks, candies, snacks and fast food were excluded. The diet was applied for one year under the nutritional practices of childhood obesity treatment program. Because of the practice, a decrease in BMI values was observed in both girls and boys [23].

\section{Inability to manage energy intake}

Children are insufficient to control the energy they intake. However, children exposed to malnutrition can be conditioned for excessive nutrient intake independent of their hunger and satiety feelings [20]. In addition, as children learn by observing their surroundings, food intake in large portions pushes children to food intake in large portions too. In addition, children who are constantly fed with large portions or excessive feeding, may be overweight and obese in time due to developing habits to eat large portions or eating excessively. The educational status and orientation of the parents affects the child's eating habits and the prevalence of obesity in children [5,19]. Fast food consumption of children that are oriented by their parents actively is lower [13].

Weker [24] found that obese children $(n=236)$ intended to investigate the effects of nutritional factors on obesity and found that familial factors and environmental conditions had an effect on childhood obesity. In addition, a statistically significant correlation was found between obesity and education level of mothers and obesity frequency of fathers [24]. 
Zhao et al. (2017) aimed to determine obesity frequency and fast food consumption of children in their study. And they found that children of college or university graduated mothers less fast food and had a lower prevalence of obesity [22].

In a study conducted by reaching 13396 adolescents (aged from 67 to 11 years) from 6781 families, the effect of maternal education, purchasing power, and status at work on adolescents' nutritional behavior was examined. It has been determined that the education status of the mother and the status of the workplace are related to the nutritional status and that the children of these mothers have a higher average height length according to their age. In addition, even if they had BMI differences, it was observed that the difference in BMI between boys and girls was lower in children of mothers with higher educational status. Also, fast-food consumption rates found to be higher in boys whose mothers had higher education levels [25].

\section{Nutritional behaviours of the family}

Another factor that is effective on the nutritional behaviors of children is the nutritional behavior and nutritional attitudes of the family [5-26]. The role of family in childhood obesity, a major public health problem, is great. For this reason, families should be reached, and education should be provided to families to be able to have the right nutrition practices [27].

Family-related factors such as the number of people in the family, the child having younger brother, and the mother's nutrition habits are known to affect the health of the child [28]. Family-oriented lifestyle modification practices cause significant changes in lifestyle and nutrition habits among obese children. In addition, this practice reduces the desire to eat excessively in children and affects the blood parameters positively [17].

In a study of how families' nutrition and activities affected children, it was observed that unhealthy nutritional behaviors, habits and inactivity of the families affected their children's behavior. It was also found that families with unhealthy eating habits and behaviors had less activity and that children of these families exhibited similar behaviors and consumed more foods that has high energy, saturated fat and salt. It was also determined that the proportion of overweight and obese children was higher among these children. As a result of the study, it was concluded that the family has a great influence on the child's nutritional and physical activity behaviors and has a serious role in the development of childhood obesity [29].

In a study that investigated effects of family's nutritional status on children's nutritional status, 698 children (6-12 years) and their families were included. The results of the study showed that the eating habits and energy intake of the families and the children were similar, and the relationship was statistically significant [26].

The fact that either or both parents of a child is/are being obese is a sign that the child will also tend to become obese $[5,12]$. In particular, these parents may be able to restrict the food intake of their children because they are concerned that their children will be fat. And as a result, they may cause their children to get more nutrients by creating an adverse reaction. Especially if the mother and father do not exhibit the right nutritional behavior themselves, the situation gets serious. To control children's energy intake and nutritional behaviors, parents either use food as a reward or restrict their children's access to highnutrient energy content. In both cases, children want to consume more of these foods, resulting in the opposite outcome [12,20]. Other factors, such as eating together meals, number of meals, meal time, etc., are alsoeffective in childhood obesity. Children who consume meals together with their family eat healthier [19,30].

In a study of the relationship between the frequency of meal consumption of adolescents and their families 'and their prevalence of obesity, 890 adolescents (12-13 years) were included. Obesity prevalence was found to be significantly higher in girls who consumed evening meals alone in terms of the data obtained in the study, but it was found that there was no meaningful relationship in boys [31].

In another study, the relationship between the anxiety of the parents on the body weight of their children, the food intake of children, the food at home, the meal times of family, and the nutrition practices of families on their children were examined. Within the scope of the study, 1448 adolescents (12-15 years) and their families were reached. Twelve percent of the families said they thought their children were fat, while $27 \%$ said they were concerned about the weight of their children. Children of disconcerting families reported that they consumed less energy-high foods, having less of these foods in their homes, and that their families listened less frequently while shopping and cooking compared to other children. However, it has been determined that anxious families are not found to have or consume more fruits and vegetables at home and did not plan mealtimes [32].

When treatment of childhood obesity and reduction of prevalence is aimed to improve family related factors maternal and paternal involvement is included. But there is an important role for grandparents in the nutritional status and behaviors of children [33]. In a study conducted, it was determined that grandparents had an important effect on children's nutritional status and behaviors and physical activity levels as well as their parents $[33,34]$.

\section{Food types that kept at home}

The presence of unhealthy, energy-rich and nutritious foods at home causes children to consume more of this type of food and develop nutritional habits of consuming higher energy foods over the years to come [35].

Contradictorily, the prevalence of obese children is higher in lower income families compared to families with higher income levels. The reason for this situation is related to purchase and consumption of foods high in energy content in low-income families [5-19].

In a study conducted with the participation of 1157 adolescents in which factors affecting childhood obesity were examined, participants were asked about height, body weight, socio-demographic characteristics, nutrient intake, and physical activity behaviors. According to the data from the study overweight and obesity rates were related with in low and high-income families, mothers working outside house, those whose both parents were obese, those who spent a long time against TV, those who had high energy intake, those who had low physical activity, those who had insufficient powder intake, those who skip breakfasts more and those who have low and high fat intake. It is also found that as the energy intake increases the obesity rate increases and as the vegetable consumption increases fiber intake increases, obesity rate decreases. Adolescents with moderate economic status were found to have lower risk of being overweight / obese than adolescents with low and high socioeconomic status [36].

In study conducted to assess food safety, 55 pairs of mothers and children were included and $65.4 \%$ of mothers and $43.6 \%$ of children stated that food safety and foods stored at house were insufficient. Statistically significant relationships were found between food safety, 
stored food at house and fast food consumption of mothers and children, eating as a family, conscious eating and binge eating behaviors. At the end of the study, it was decided that the food at home and the safety of these foods affected the eating habits of the children [37].

In a study conducted, it was determined that the families struggled to have healthy eating behaviors for themselves and their children, but the home environment and environmental factors did not support this [27]. In another study of the relationship between home nutrition environment and childhood obesity, 669 children and their families were reached, and nutritional status and body weights were examined. As a result of the study, it was found that the consumption of vegetables and fruits of children was significantly higher when their parents set an example and they are supported, but when there were unhealthy foods at home, vegetable and fruit consumption of children were found to be significantly lower [38].

\section{Maternal nutritional status during pregnancy and breastfeeding}

Although the mechanisms behind maternal nutritional status during pregnancy and breastfeeding and childhood obesity are not clearly explained, maternal feeding is thought to be directly related to childhood obesity [12-20]. In particular, factors such as the cultural structure of the population mother lives in, the economic level of the family affects the mother's nutritional status and behaviors as well as the nutritional status and behavior of the child during pregnancy, breastfeeding and growth periods [19].

In a study in which the effects of pregnancy and lactation-related factors on obesity in children were examined, body weights were evaluated according to the height of 690 children aged 0-12 months. It was determined that $12.9 \%$ of the children were overweight and $9.7 \%$ were obese. It was found that as the BMIs of the parents and the weight gain in pregnancy increases, the weight of the children increases. And it was determined that the first six months of breastfeeding had protective effects on obesity in these children. As a result of the study, breastfeeding and weight gain in pregnancy are affecting childhood obesity as well as genetic factors and they are modifiable factors directly effecting childhood obesity [39].

\section{Conclusion}

Childhood obesity is a health problem that affects children's health negatively as physiologically, socially and psychologically, and needs to be intervened and treated as soon as possible in order to improve their health. It is also a risk factor for adulthood obesity and many chronic diseases in adults.

The policies to be followed for treatment should include national and international approaches. However, when the reasons for childhood obesity are taken into consideration, requirement of multidisciplinary approaches emerges. In addition to this, it is also very important to improve the environmental conditions, give education and to provide lifestyle changes.

One of the main causes of childhood obesity is nutrition. And factors affecting nutritional status and nutrition-related factors. Dietitians have a great duty in evaluation and improvement of these factors. And they should be able to implement correct approaches and nutritional practices during diagnosis and assessment of childhood obesity. Dieticians should actively take part in epidemiologic studies, national or international politics and practices to improve this situation.

\section{References}

1. World Health Organization (2018) Child Growth Standards.

2. UNICEF-WHO-The World Bank Joint Child Malnutrition Estimates (2018) Levels \& Trends in Child Malnutrition.

3. World Health Organization (2018) Adolescent Health. http://www.who.int/topics/ adolescent health/en/

4. Rees JM, Neumark-Sztainer D (1999) Improving the nutritional health of adolescentsposition statement-society for adolescent medicine. Journal of Adolescent Health 24: 461-462.

5. Mangels R, Stallman I, Davis EH, Mofidi S, Lee AR, et al. (2015) Life Cycle Nutrition an Evidence-Based Approach. Eldestein S, Edn, Jones \& Barlett Learning, Canada 247-295.

6. Hong SA, Peltzer K (2017) Dietary behaviour, psychological well-being and mental distress among adolescents in Korea. Child and Adolescent Psychiatry and Mental Health 11: 1-12.

7. Saikia D, Ahmed SJ, Saikia H, Sama R (2016) Overweight and obesity in early adolescents and its relation to dietary habit and physical activity: A study in Dibrugarh town. Clinical Epidemiology and Global Health 4: 22-28.

8. World Health Organization (2018) Child Growth Standards

9. Mekonnen T, Tariku A, Abebe SM (2018) Overweight/obesity among school aged children in Bahir Dar City: Cross sectional study. Italian Journal of Pediatric 44: 1-8.

10. School Ages of Children in Turkey (6-10 Age Group) tracked the growth of the (TOÇB) Project Research Report. T. C. Ministry of Health TSH General Directorate, Hacettepe University Faculty of Health Sciences Department of Nutrition and Dietetics, TC Ministry of Education, Department of Health Affairs. Ankara, 2011.

11. Turkey Childhood Obesity Research (CON of-ENG) 2016 Ministry of Health, Public Health Agency of Turkey, Ministry of National Education, Hacettepe University, Ministry of Health, Ankara, 2017.

12. Wardlaw GM, Hampl JS (2007) Perspectives in Nutrition. Edn 7, Mc Graw Hill, New York.

13. Lwin MO, Malik SM, Ridwan H, Sum CSA (2017) Media exposure and parenta mediation on fast-food consumption among children in metropolitan and suburban Indonesian. Asia Pacific Journal of Clinical Nutrition 26: 899-905.

14. Sarkar SR, Sil SK (2016) Nutritional status of rural Bengali adolescent boys of tripura Indian Journal Pediatrics 83: 356.

15. Erkan T (2011) Ergenlerde beslenme. Türk Pediatri Arsivi 46: 49-53.

16. Burkhart SJ, Coad J (2010) Assessment of nutritional knowledge and food skills in talented adolescent athletes. New Zealand: Massey University Institute of Food, Nutrition \& Human Health

17. Cohen TR, Hazell TJ, Vanstone CA, Rodd C, Weiler HA (2018) Changes in eating behavior and plasma leptin in children with obesity participating in a family-centered lifestyle intervention. Appetite 125: 81-89.

18. Mikkila V, Vepsalainen H, Saloheimo T, Gonzales SA, Meisel JD, et al. (2015) An international comparison of dietary patterns in 9-11-year-old children. International Journal of Obesity Supplements 5: 17-21.

19. Insel P, Turner RE, Ross D (2007) Nutrition. Edn, Jones and Bartlett Publishers Inc, Canada.

20. Bender DA (2014) Introduction to Nutrition and Metabolism. Edn 5, CRC Press, United States.

21. Ma R, Castellanos DC, Bachman J (2016) Identifying factors associated with fast food consumption among adolescents in Beijing China using a theory-based approach. Public Health 136: 87-93.

22. Zhao Y, Wang L, Xue H, Wang H, Wang Y (2017) Fast food consumption and its associations with obesity and hypertension among children: results from the baseline data of the Childhood Obesity Study in China Mega-cities. BMC Public Health 17: 933. [Crossref]

23. Trier C, Fonvig CE, Bøjsøe C, et al. (2016) No influence of sugar, snacks and fast food intake on the degree of obesity or treatment effect in childhood obesity. Pediatr Obes 11: 506-512. [Crossref]

24. Weker H (2006) Simple obesity in children. A study on the role of nutritional factors. Med Wieku Rozwoj 10: 3-191. [Crossref] 
25. Kunto YS, Bras H (2017) Women's Empowerment and Gender Inequality in Adolescent Nutritional Status: Evidence From The Indonesian Family Life Survey. Journal of Biosocial Science 1-26.

26. Robson SM, Couch SC, Peugh JL, Glanz K, Zhou C, et al. (2016) Parent Diet Quality and Energy Intake Are Related to Child Diet Quality and Energy Intake. J Acad Nutr Diet 116: 984-990. [Crossref]

27. Luesse HB, Paul R, Gray HL, Koch P, Contento I, et al. (2018) Challenges and facilitators to promoting a healthy food environment and communicationg effectively with parents to improve food behaviors of school children. Maternal and Child Health Wournal $1-10$

28. Dasgupta K, Solomon KT (2018) Family size effects on childhood obesity: Evidence on the quantity-quality trade-off using the NLSY. Econ Hum Biol 29: 42-55. [Crossref]

29. Liu J, Hay J, Faught BE, Wade T, Cairney J, et al. (2012) Family eating and activity habits, diet quality and pre-adolescent overweight and obesity. Public Health 126: 532-534.

30. Bussiek PV, De Poli C, Bevan G (2018) A scoping review protocol to map the evidence on interventions to prevent overweight and obesity in children. BMJ Open 8: e019311. [Crossref]

31. Shirasawa T, Ochiai H, Yoshimoto T, Matoba M, Sunaga Y, et al. (2018) Effects of eating dinner alone on overweight in Japanese adolescents: a cross-sectional survey. BMC Pediatrics 18: 1-6.

32. MacFarlane A, Crawford D, Worsley A (2010) Associations between parental concern for adolescent weight and the home food environment and dietary intake. Journal of Nutrition Education and Behaviour 42: 152-160.
33. Bell LK, Perry RA, Prichard I (2018) Exploring grandparent' roles in young children's lifestyle behaviors and the prevention of childhood obesity: an Australian Perspective. Journal of Nutrition Education and Behavior 1-7.

34. Eli K, Howell K, Fisher PA, Nowicka P (2016) A question of balance: Explaining differences between parental and grandparental perspectives on preschoolers' feding and physical activity. Social Science Medicine 154: 28-35.

35. Fernando NNT, Campbell KJ, McNaughton SA, Zheng M, Lacy KE (2018) Predictors of dietary energy density among preschool aged children. Nutrients 10: 1-17.

36. Hatami M, Taib MNM, Jamaluddin R, Saad HA, Djazayery A, et al. Dietary factors as the majör determinants of overweight and obesity among Iranian adolescents. A crosssectional study. Appetite 82: 194-201

37. Bruening M, Lucio J, Brennhofer S (2017) Mother and adolescent eating in the contex of food insecurity: findings from urban public housing. Maternal and Child Health Journal 21: 1911-1917.

38. Couch SC, Glanz K, Zhou C, Sallis JF, Saelens BE (2014) Home food environment in relation to children's diet quality and weight status. J Acad Nutr Diet 114: 1569-1579. [Crossref]

39. Hazrati S, Wong WSW, Huddleston K, Yui Y, Gilchrist N, et al. (2018) Clinical, Social, and Genetic Factors Associated with Obesity at 12 Months of Age. J Pediatr 196: 175 181. [Crossref]

Copyright: (C2018 Çirak O. This is an open-access article distributed under the terms of the Creative Commons Attribution License, which permits unrestricted use, distribution, and reproduction in any medium, provided the original author and source are credited. 